HEP-TH/0604069

NORDITA-2006-11

\title{
A Universality Test of the Quantum String Bethe Ansatz
}

\author{
L. Freyhult ${ }^{1}$ and C. Kristjansen ${ }^{2}$ \\ NORDITA, \\ Blegdamsvej 17, DK-2100 Copenhagen Ø
}

\begin{abstract}
We show that the quantum corrected string Bethe ansatz passes an important universality test by demonstrating that it correctly incorporates the non-analytical terms in the string sigma model one-loop correction for rational three-spin strings with two out of the three spins identical. Subsequently, we use the quantum corrected string Bethe ansatz to predict the exact form of the non-analytic terms for the generic rational three-spin string.
\end{abstract}

\footnotetext{
${ }^{1}$ freyhult@nordita.dk

${ }^{2}$ kristjan@nbi.dk
} 


\section{Introduction}

The discovery of integrable structures governing the spectrum of $\mathcal{N}=4$ SYM [1-3] as well as that of type IIB superstrings on $A d S_{5} \times S^{5}[4,5]$ has shifted the focus away from the dilatation operator of the gauge theory respectively the string Hamiltonian towards that of the S-matrices of the underlying integrable models [6].

On the $\mathcal{N}=4 \mathrm{SYM}$ side the relevant $S$-matrix has been determined in perturbation theory to three-loop order in certain sectors [6] and a conjecture for an all sector, all loop $S$-matrix exists [7]. In addition, S-matrix techniques have proved useful for the study of various generalizations of the gauge theory $[8,9]$.

Due to our current inability to quantize the string theory on $A d S_{5} \times S^{5}$ the S-matrix underlying the integrable structures on the string theory side is less well understood. Based on input from pp-wave physics as well as various semi-classical analyses it has been proposed that the string theory $S$-matrix differs from the gauge theory one only by a phase factor, expressible in terms of certain conserved charges $[10,6,7]$, see also $[11,12]$.

$$
S^{\text {string }}\left(p_{k}, p_{j}\right)=e^{2 i \theta\left(p_{k}, p_{j}\right)} S^{\text {gauge }}\left(p_{k}, p_{j}\right) .
$$

The most general possible form of the phase consistent with integrability and respecting the symmetries of $\mathcal{N}=4 \mathrm{SYM}$ reads [8]

$$
\theta\left(p_{k}, p_{j}\right)=\sum_{r=2}^{\infty} \sum_{s=r+1}^{\infty} c_{r, s}(\lambda)\left(\frac{\lambda}{16 \pi^{2}}\right)^{\frac{r+s-1}{2}}\left(q_{r}\left(p_{k}\right) q_{s}\left(p_{j}\right)-q_{s}\left(p_{j}\right) q_{r}\left(p_{k}\right)\right)
$$

where $c_{r, s}=0$ unless $r+s$ is odd. Here, $q_{r}\left(p_{k}\right)$ is the contribution to the $r$ 'th conserved charge from the $k$ 'th excitation and $\lambda$ is the 't Hooft coupling constant.

With $c_{r, s}=\delta_{s, r+1}$ the above string $S$-matrix gives rise to the correct continuum Bethe equations describing the motion of classical strings on $A d S_{5} \times S^{5}$ [13-18] and describes correctly strings in the BMN-limit [19] and the near BMN-limit [20]. In particular, this string $S$-matrix accounts for the observed three-loop discrepancy between gauge theory and string theory [21-23]. In all of these instances the string energies are analytic in $\lambda$. String sigma model one-loop corrections, however, lead to half-integer powers of $\lambda$ appearing in the expressions for energies of spinning strings [24-26]. It was suggested that these could be accommodated in the string Bethe ansatz by assuming $c_{r, s}$ to have an expansion in half-integer inverse powers of $\lambda$, i.e. [26]

$$
c_{r, s}(\lambda)=\delta_{s, r+1}+\delta c_{r, s} \frac{1}{\sqrt{\lambda}}+\mathcal{O}\left(\frac{1}{\lambda}\right) .
$$

Here, the first $1 / \sqrt{\lambda}$ correction term should be enough to account for the string sigma model one-loop correction. This idea was tested for strings dual to gauge theory operators in respectively the $\mathfrak{s u}(2)$ sector at half-filling and the $\mathfrak{s l}(2)$ sector $[26,27]$. Whereas the $\mathfrak{s u}(2)$ case did not fix the corrections to the coefficients $c_{r, s}$ uniquely, the study of the $\mathfrak{s l}(2)$ sector did and lead to a conjecture for $\delta c_{r, s}[27]$. As the quantum string Bethe ansatz conjecture implies that the dressing factor should be universal [7], it is important that the coefficients $\delta c_{r, s}$ be the same for all sectors. In the present paper we show that 
the quantum string Bethe ansatz passes an important universality test by demonstrating that string sigma model one loop corrections in the $\mathfrak{s u}(3)$ sector, i.e. for strings with three large angular momenta on $S^{5} \subset A d S_{5} \times S^{5}$, also uniquely fix the $\delta c_{r, s}$ and that to values coinciding with the conjectured ones.

The paper is organised as follows. In section 2 we formulate the quantum corrected string Bethe ansatz for strings with three large angular momenta on $S^{5}$ and use it to derive the first non-analytical contributions to energies of rational three-spin strings. Subsequently, in section 3 we determine the non-analytical corrections via a one-loop string sigma model calculation for the case of a three-spin string with two out of the three spins identical. Matching the two results we in section 4 uniquely determine the coefficients $\delta c_{r, s}$ in the quantum corrected string Bethe ansatz. We furthermore use these results to predict the string sigma model one-loop corrections for rational three spin strings with arbitrary values of the three angular momenta and the two possible winding numbers. Finally, section 5 contains our conclusions.

\section{Non-analytic corrections from the string Bethe ansatz}

The conjectured string Bethe ansatz [7] does not in general allow one to treat the $\mathfrak{s u}(3)$ sector as closed but rather demands that one considers the larger $\mathfrak{s u}(2 \mid 3)$ sub-sector. However, as we are interested in studying spinning strings we will always be working in the thermodynamical limit where the $\mathfrak{s u}(3)$ sector is effectively closed [28].

In the thus effectively closed $\mathfrak{s u}(3)$ sector one has two types of Bethe roots with filling fractions $\alpha$ and $\beta$ related to the angular momentum labels of the string by

$$
\left(J_{1}, J_{2}, J_{3}\right)=J(1-\alpha, \alpha-\beta, \beta),
$$

where $J$ is the length of the spin chain. The thermodynamical limit of the proposed string Bethe equations read

$$
\begin{aligned}
& V_{1}(\varphi)=2 f \frac{\rho_{1}\left(\varphi^{\prime}\right)}{\varphi-\varphi^{\prime}}-\int \frac{\rho_{2}\left(\varphi^{\prime}\right)}{\varphi-\varphi^{\prime}} \\
& V_{2}(\varphi)=2 f \frac{\rho_{2}\left(\varphi^{\prime}\right)}{\varphi-\varphi^{\prime}}-\int \frac{\rho_{1}\left(\varphi^{\prime}\right)}{\varphi-\varphi^{\prime}}
\end{aligned}
$$

with $\rho_{1}(\varphi)$ and $\rho_{2}(\varphi)$ being the densities of Bethe roots with filling fractions $\alpha$ and $\beta$ respectively. In the case of rational three spin strings the potentials are

$$
\begin{aligned}
& V_{1}(\varphi)=2 \pi n+\frac{1}{\sqrt{\varphi^{2}-4 \omega^{2}}}-\sum_{r, s} \omega^{r+s-1} c_{r, s}(\lambda)\left(q_{r}(\varphi) Q_{s}-Q_{r} q_{s}(\varphi)\right), \\
& V_{2}(\varphi)=2 \pi m,
\end{aligned}
$$

where $m$ and $n$ are integers and where the charges are given by

$$
q_{r}(\varphi)=\frac{1}{\sqrt{\varphi^{2}-4 \omega^{2}}} \frac{1}{\left(\frac{1}{2} \varphi+\frac{1}{2} \sqrt{\varphi^{2}-4 \omega^{2}}\right)^{r-1}}, \quad Q_{r}=\int d \varphi^{\prime} \rho_{1}\left(\varphi^{\prime}\right) q_{r}\left(\varphi^{\prime}\right) .
$$


Here we have used the notation

$$
\omega=\frac{1}{4 \pi \mathcal{J}}=\frac{\sqrt{\lambda}}{4 \pi J} .
$$

The string Bethe equations have to be supplemented by the level matching condition

$$
Q_{1}=2 p \pi, \quad p \in \mathbb{Z}
$$

We are interested in determining the leading non-analytical contributions to the string energy, i.e.

$$
\delta \mathcal{E}_{\text {non-analytic }}=\left(Q_{2}\right)_{\text {non-analytic }} .
$$

It is easy to see that with the ansatz (3) for the coefficients $c_{r, s}$ the corrections $\delta c_{r, s}$ will for finite $\omega$ be of the same order as the leading finite size effects studied in [29] (and for the $\mathfrak{s u}(2)$ and $\mathfrak{s l}(2)$ sector in [30-32]). As the leading finite size corrections are analytical in $\lambda$ we, however, do not need to worry about mixing of the two effects and can safely ignore the finite size corrections.

Using a similar trick as in [29] we can turn the above relations into a quadratic and a cubic equation. Let us introduce the resolvents

$$
G_{1}(\varphi)=\int \frac{\rho_{1}\left(\varphi^{\prime}\right)}{\varphi-\varphi^{\prime}}, \quad G_{2}(\varphi)=\int \frac{\rho_{2}\left(\varphi^{\prime}\right)}{\varphi-\varphi^{\prime}} .
$$

which have the following behaviour as $\varphi \rightarrow \infty$

$$
G_{1}(\varphi) \rightarrow \frac{\alpha}{\varphi}, \quad G_{2}(\varphi) \rightarrow \frac{\beta}{\varphi} .
$$

The quadratic and cubic equations can then be written as

$$
\begin{aligned}
& G_{1}^{2}(\varphi)+G_{2}^{2}(\varphi)-G_{1}(\varphi) G_{2}(\varphi)-V_{2}(\varphi) G_{2}(\varphi)-V_{1}(\varphi) G_{1}(\varphi) \\
& +\operatorname{Res}_{x=0}\left(\frac{G_{1}(x) V_{1}(x)}{\varphi-x}\right)=0, \\
& G_{2}^{2}(\varphi) G_{1}(\varphi)-G_{1}^{2}(\varphi) G_{2}(\varphi)-V_{2}(\varphi) G_{2}^{2}(\varphi)+V_{2}^{2}(\varphi) G_{2}(\varphi)+V_{1}(\varphi) G_{1}^{2}(\varphi)-V_{1}^{2}(\varphi) G_{1}(\varphi) \\
& -\operatorname{Res}_{x=0}\left(\frac{G_{1}^{2}(x) V_{1}(x)}{\varphi-x}\right)+\operatorname{Res}_{x=0}\left(\frac{G_{1}(x) V_{1}^{2}(x)}{\varphi-x}\right)=0 .
\end{aligned}
$$

Let us denote the negative moments of the root distributions as $\mathcal{Q}_{i}$ and $\mathcal{P}_{i}$, i.e.

$$
G_{1}(\varphi)=-\sum_{i=0}^{\infty} \mathcal{Q}_{i+1} \varphi^{i}, \quad G_{2}(\varphi)=-\sum_{i=0}^{\infty} \mathcal{P}_{i+1} \varphi^{i} .
$$

From simply integrating over $\varphi$ in (5) we find

$$
Q_{1}=2 \pi(n \alpha+m \beta) .
$$

Expanding the cubic and quadratic equation for large and small values of $\varphi$ allows us to determine the resolvents perturbatively in the coupling, $\omega$. The cubic equation at 
infinity gives $Q_{2}$ in terms of the moments $\mathcal{Q}_{i}$ and $\mathcal{P}_{i}$. (The quadratic equation at infinity is redundant as it is equivalent to the momentum condition.) The moments are obtained by first eliminating $Q_{1}$ using the momentum constraint and next using the quadratic and cubic equation interchangeably to determine $\mathcal{P}_{i}$ and $\mathcal{Q}_{i}$. The algorithm obviously works for any values of $\alpha, \beta, m$ and $n$, i.e. for any rational three-spin string, but for simplicity we shall present the result only for the case where two out of the three angular momenta of the string are equal ${ }^{3}$. Thus, let us specialize to the case ${ }^{4}$

$$
\beta=\frac{\alpha}{2}, \quad n=-\frac{m}{2} \equiv k,
$$

where $k$ is now the single remaining winding number of the string. Using the above discussed expansions of (15) and (16) we find the following non-analytic contribution to the energy ${ }^{5}$

$$
\begin{aligned}
& \delta \mathcal{E}_{\text {non-analytic }}=\frac{1}{16 \mathcal{J}^{5}} \delta c_{2,3} k^{6} \alpha^{2}(3 \alpha-2) \\
& +\frac{1}{64 \mathcal{J}^{7}} k^{8} \alpha^{2}\left(\left(10-23 \alpha+8 \alpha^{2}\right) \delta c_{2,3}+\left(2-7 \alpha+6 \alpha^{2}\right) \delta c_{3,4}-2\left(1-5 \alpha+5 \alpha^{2}\right) \delta c_{2,5}\right) \\
& +\frac{1}{256 \mathcal{J}^{9}} k^{10} \alpha^{2}\left(\delta c_{2,3}\left(-42+131 \alpha-64 \alpha^{2}-24 \alpha^{3}\right)+\delta c_{3,4}\left(-14+65 \alpha-88 \alpha^{2}+31 \alpha^{3}\right)\right. \\
& +\delta c_{2,5}\left(14-94 \alpha+154 \alpha^{2}-63 \alpha^{3}\right)+\delta c_{2,7}\left(-2+21 \alpha-56 \alpha^{2}+42 \alpha^{3}\right) \\
& \left.+\delta c_{3,6}\left(2-15 \alpha+32 \alpha^{2}-21 \alpha^{3}\right)+\delta c_{4,5}\left(-2+14 \alpha-30 \alpha^{2}+20 \alpha^{3}\right)\right)+\ldots(20)
\end{aligned}
$$

\section{Non-analytic corrections from a string sigma model one loop calculation}

The string sigma model one-loop correction has only been explicitly worked out for two classes of classical string solutions. One class is rational two-spin strings with one large momentum on $S^{5}$ and one on $A d S_{5}$, dual to operators in the $\mathfrak{s l}(2)$ sector of the gauge theory [33]. The other class is rational three spin strings with three large angular momenta on $S^{5}$, two of them being identical [34-36]. (For partial results on the generic three-spin case, see $[37,38]$.) In the present paper we shall consider the latter case denoting the three spins as $\left(\mathcal{J}_{1}, \mathcal{J}_{2}, \mathcal{J}_{2}\right)$ and the sum of these as $\mathcal{J}$. (When $\mathcal{J}_{1}$ vanishes this case reduces to two-spin strings dual to operators in the $\mathfrak{s u}(2)$ sector at half-filling.) The one-loop energy receives contributions from fluctuations in the bosonic (B) as well as the fermionic (F) fields both in the $S^{5}$ and in the $A d S_{5}$ part of the space and takes the form $[35,36]$

$$
\delta E=\frac{1}{2 \kappa}\left(\sum_{n \in \mathbb{Z}} \omega_{n}^{B}-\sum_{r \in \mathbb{Z}+1 / 2} \omega_{r}^{F}\right),
$$

\footnotetext{
${ }^{3}$ For an example of the complexity of the general result, see section 4

${ }^{4}$ This choice allows us to get the result for all possible three-spin strings with two out of three angular momenta identical, see detailed discussion in [29].

${ }^{5}$ The calculation can be taken much further but for simplicity we limit ourselves to presenting only the first three terms.
} 
where $\kappa$ relates world-sheet and the $A d S_{5}$ time as $t=\kappa \tau$. Here $\omega$ is the absolute value of the frequencies ${ }^{6}$. The contributions to $\omega^{B}$ from fluctuations in the $S^{5}$ directions are given by the roots of the polynomial

$$
\begin{aligned}
& B_{8}(\Omega)=\Omega^{4}+\Omega^{3}\left(-8 k^{2}-4 n^{2}+20 k^{2} q-8 \kappa^{2}\right)+\Omega^{2}\left(16 k^{4}+8 k^{2} n^{2}+6 n^{4}-80 k^{4} q\right. \\
& \left.\quad-36 k^{2} n^{2} q+96 k^{4} q^{2}+32 k^{2} \kappa^{2}+16 n^{2} \kappa^{2}-80 k^{2} q \kappa^{2}+16 \kappa^{4}\right)+\Omega\left(-32 k^{4} n^{2}+8 k^{2} n^{4}\right. \\
& \left.\quad-4 n^{6}+96 k^{4} n^{2} q+12 k^{2} n^{4} q-96 k^{4} n^{2} q^{2}-32 k^{2} n^{2} \kappa^{2}-8 n^{4} \kappa^{2}+48 k^{2} n^{2} q \kappa^{2}\right) \\
& \quad+16 k^{4} n^{4}-8 k^{2} n^{6}+n^{8}-16 k^{4} n^{4} q+4 k^{2} n^{6} q
\end{aligned}
$$

where $\Omega=\omega^{2}$ and

$$
\kappa=\sqrt{\nu^{2}+2 q}, \quad q=\frac{\alpha \mathcal{J}}{\sqrt{\nu^{2}+1}}
$$

and $\nu$ is given by

$$
(\nu-\mathcal{J}(1-\alpha)) \sqrt{1+\nu^{2}}-\alpha \mathcal{J} \nu=0 .
$$

We have introduced $\alpha$ to denote the fraction $2 \mathcal{J}_{2} / \mathcal{J}$. The strings we consider are localized at the center of $A d S_{5}$ and the bosonic frequencies associated with fluctuations in that part of the space therefore take the simple form

$$
\omega_{A d S}=\sqrt{n^{2}+\kappa^{2}}
$$

The fermionic frequencies mix fluctuations in $S^{5}$ and $A d S_{5}$ and are given by the roots of the polynomial

$$
\begin{aligned}
F_{8}( & \Omega)=2 \Omega^{4}+\Omega^{3}\left(-8 k^{2}-12 \kappa^{2}-8 r^{2}+20 k^{2} q\right) \\
& +\Omega^{2}\left(12 k^{4}+28 k^{2} \kappa^{2}+18 \kappa^{4}+8 k^{2} r^{2}+28 \kappa^{2} r^{2}+12 r^{4}\right. \\
& \left.-52 k^{4} q-64 k^{2} \kappa^{2} q-36 k^{2} r^{2} q+59 k^{4} q^{2}\right) \\
& +\Omega\left(-8 k^{6}-20 k^{4} \kappa^{2}-20 k^{2} \kappa^{4}-8 \kappa^{6}+8 k^{4} r^{2}+8 k^{2} r^{2} \kappa^{2}-20 \kappa^{4} r^{2}+8 k^{2} r^{4}-20 \kappa^{2} r^{4}\right. \\
& -8 r^{6}+44 k^{6} q+80 k^{4} \kappa^{2} q+44 k^{2} \kappa^{4} q-24 k^{4} r^{2} q+32 k^{2} \kappa^{2} r^{2} q+12 k^{2} r^{4} q-78 k^{6} q^{2} \\
& \left.-79 k^{4} \kappa^{2} q^{2}+2 k^{4} r^{2} q^{2}+45 k^{6} q^{3}\right) \\
& +2 k^{8}+4 k^{6} \kappa^{2}+2 k^{4} \kappa^{4}-8 k^{6} r^{2}-4 k^{4} \kappa^{2} r^{2}-4 k^{2} \kappa^{4} r^{2}+12 k^{4} r^{4}-4 k^{2} \kappa^{2} r^{4}+2 \kappa^{4} r^{4} \\
& -8 k^{2} r^{6}+4 \kappa^{2} r^{6}+2 r^{8}-12 k^{8} q-16 k^{6} \kappa^{2} q-4 k^{4} \kappa^{4} q+28 k^{6} q r^{2}+16 k^{4} \kappa^{2} r^{2} q \\
& +4 k^{2} \kappa^{4} r^{2} q-20 k^{4} r^{4} q+4 k^{2} r^{6} q+27 k^{8} q^{2}+21 k^{6} \kappa^{2} q^{2}+2 k^{4} \kappa^{4} q^{2}-30 k^{6} r^{2} q^{2} \\
& -11 k^{4} \kappa^{2} r^{2} q^{2}+11 k^{4} r^{4} q^{2}-27 k^{8} q^{3}-9 k^{6} \kappa^{2} q^{3}+9 k^{6} r^{2} q^{3}+81 / 8 k^{8} q^{4} .
\end{aligned}
$$

The expression for the one-loop energy shift (21) is convergent, the constituent bosonic and fermionic sums are, however, divergent. Rearranging the sums taking into account that they individually diverge (21) can be rewritten as in [36]

$$
\delta E=\frac{1}{2 \kappa}\left[2+\sum_{n \in \mathbb{Z}}\left(\omega_{n}^{B}-\frac{1}{2}\left(\omega_{n+1 / 2}^{F}+\omega_{n-1 / 2}^{F}\right)\right)\right] .
$$

\footnotetext{
${ }^{6}$ This is not true in the general case, however as long as half of the frequencies are negative and the other half positive this holds. See [39,33] for details.
} 
The sum is again convergent. However, the individual terms in the $1 / \mathcal{J}$-expansion are divergent. A way to get around an artificial regularisation of the sum is to split the one-loop correction as follows [26,40]

$$
\delta E=\sum_{n=-\infty}^{\infty} e_{\text {reg }}^{\text {sum }}(n)+\mathcal{J} \int_{-\infty}^{\infty} d x e_{\text {reg }}^{\text {int }}(x),
$$

where the last part is equal to the divergent part of the expanded sum and encodes the non-analytic contribution. It can be computed, following the procedure in [26] ,by first making the rescaling $n=\mathcal{J} x$, then expanding in $1 / \mathcal{J}$ and finally integrating over the regular part of the obtained expression. The computations can be simplified setting the winding number, $k$, to one. It is always possible to, in the end, restore it by rescaling the variables as

$$
n \rightarrow \frac{n}{k}, \quad \nu \rightarrow \frac{\nu}{k} \quad \text { and } \quad \omega \rightarrow \frac{\omega}{k}
$$

Setting $k=1$, rescaling $n$ and expanding we find

$$
\begin{aligned}
e^{i n t}( & x)=-\frac{1}{\mathcal{J}^{2}} \frac{1}{2\left(1+x^{2}\right)^{3 / 2}} \\
& -\frac{1}{\mathcal{J}^{4}} \frac{16 \alpha^{2}-x^{2}\left(13+12 \alpha-80 \alpha^{2}\right)+x^{4}\left(52-48 \alpha+64 \alpha^{2}\right)+24 x^{6} \alpha}{32 x^{2}\left(1+x^{2}\right)^{7 / 2}} \\
& -\frac{1}{\mathcal{J}^{6}} \frac{1}{256 x^{4}\left(1+x^{2}\right)^{11 / 2}}\left(256 \alpha^{2}+64 x^{2} \alpha^{2}(21-\alpha)\right. \\
& +x^{4}\left(151-106 \alpha+2504 \alpha^{2}-64 \alpha^{3}\right)-4 x^{6}\left(453-668 \alpha-652 \alpha^{2}+312 \alpha^{3}\right) \\
& \left.+4 x^{8}\left(302-687 \alpha+940 \alpha^{2}-360 \alpha^{3}\right)+16 x^{10} \alpha\left(4+3 \alpha-12 \alpha^{2}\right)-80 x^{12} \alpha\right) \\
& -\frac{1}{\mathcal{J}^{8}} \frac{1}{8192 x^{6}\left(1+x^{2}\right)^{15 / 2}}\left(4096 \alpha^{2}\left(8-6 \alpha+3 \alpha^{2}\right)+512 x^{2} \alpha^{2}\left(463-347 \alpha+166 \alpha^{2}\right)\right. \\
+ & 256 x^{4} \alpha^{2}\left(2871-2153 \alpha+978 \alpha^{2}\right) \\
& -x^{6}\left(7565-17832 \alpha-1267952 \alpha^{2}+993152 \alpha^{3}-436224 \alpha^{4}\right) \\
+ & 8 x^{8}\left(22695-55296 \alpha+198892 \alpha^{2}-123632 \alpha^{3}+46720 \alpha^{4}\right) \\
+ & 16 x^{10}\left(-22695+54845 \alpha+4998 \alpha^{2}-25536 \alpha^{3}+14928 \alpha^{4}\right) \\
+ & 32 x^{12}\left(3026-9319 \alpha+19181 \alpha^{2}-13356 \alpha^{3}+5040 \alpha^{4}\right) \\
+ & 96 x^{14} \alpha\left(249-190 \alpha-192 \alpha^{2}+456 \alpha^{3}\right) \\
+ & \left.128 x^{16} \alpha\left(31-126 \alpha+106 \alpha^{2}+48 \alpha^{3}\right)+1792 x^{18} \alpha\left(1-2 \alpha+2 \alpha^{2}\right)\right)+\ldots
\end{aligned}
$$

We are able to go a few orders higher in the expansion (cf. eqn. (32)) but we omit those terms above as the expressions become very involved. The singular part of the above expression is

$$
\begin{aligned}
e_{\text {sing }}^{\text {int }}(x)= & -\frac{1}{\mathcal{J}^{6}}\left(\frac{1}{x^{4}} \alpha^{2}+\frac{1}{4 x^{2}} \alpha\left(1+\alpha^{2}\right)\right) \\
+ & +\frac{1}{\mathcal{J}^{8}}\left(\frac{1}{2 x^{6}} \alpha^{2}\left(8-6 \alpha+3 \alpha^{2}\right)-\frac{1}{16 x^{4}} \alpha^{2}\left(17-13 \alpha+14 \alpha^{2}\right)\right. \\
& \left.+\frac{1}{16 x^{2}} \alpha^{2}\left(3-\alpha+9 \alpha^{2}\right)\right)+\ldots
\end{aligned}
$$


Integrating the regular part and restoring the $k$-dependence we find

$$
\begin{aligned}
\mathcal{J} \int_{-\infty}^{\infty} d x e_{\text {int }}^{r e g}(x)=\frac{1}{\mathcal{J}^{5}} \frac{1}{3} k^{6} \alpha^{2}(2-3 \alpha)-\frac{1}{\mathcal{J}^{7}} \frac{1}{30} k^{8} \alpha^{2}\left(32-79 \alpha+37 \alpha^{2}\right) \\
+\frac{1}{1680 \mathcal{J}^{9}} k^{10} \alpha^{2}\left(1152-4989 \alpha+6956 \alpha^{2}-3462 \alpha^{3}\right) \\
\quad-\frac{1}{5040 \mathcal{J}^{11}} k^{12} \alpha^{2}\left(8192-55451 \alpha+134670 \alpha^{2}-139535 \alpha^{3}+50108 \alpha^{4}\right)+\ldots
\end{aligned}
$$

We notice that the integral of the two first terms in the expansion (30) vanish upon integration. A similar situation is encountered in the case of strings dual to operators in the $\mathfrak{s l}(2)$ sector $[26]$.

\section{Determination of the coefficients}

Comparing the two obtained expressions for the non-analytic contribution the string energy, (32) and (20) one can uniquely determine the coefficients $\delta c_{r, s}$. This leads to the following results

$$
\begin{aligned}
& \delta c_{2,3}=-16 / 3 \\
& \delta c_{3,4}=-48 / 3, \quad \delta c_{2,5}=-32 / 15 \\
& \delta c_{4,5}=-96 / 7, \quad \delta c_{3,6}=-80 / 21, \quad \delta c_{2,7}=-48 / 35 \\
& \delta c_{5,6}=-160 / 9, \quad \delta c_{4,7}=-16 / 3, \quad \delta c_{3,8}=-112 / 45, \quad \delta c_{2,9}=-64 / 63,
\end{aligned}
$$

which do indeed confirm the conjectured formula [27]

$$
\delta c_{r, s}=\left\{\begin{array}{cl}
-8 \frac{(r-1)(s-1)}{(r+s-2)(s-r)} & \text { if } r+s \text { odd } \\
0 & \text { if } r+s \text { even }
\end{array}\right.
$$

where it is understood that $r \geq 2$ and $s \geq r+1$. Assuming now the conjecture to be true one can, using the results of section 22 predict the non-analytical contributions to the energies of rational three-spin strings with three different angular momenta $\left(\mathcal{J}_{1}, \mathcal{J}_{2}, \mathcal{J}_{3}\right)=$ $\mathcal{J}(1-\alpha, \alpha-\beta, \beta)$ and winding numbers $(m, n)$. (Note, that according to eqns. (11) and (18) the quantity $n \alpha+m \beta$ has to be an integer.) The result for the leading correction $\operatorname{reads}^{7}$

$$
\begin{aligned}
& \delta \mathcal{E}_{\text {non-analytical }}= \\
& -\frac{1}{\mathcal{J}^{5}}\left\{\frac{1}{3} n^{6}(1-\alpha)^{3} \alpha^{3}+\frac{1}{3} m^{6}(1-\beta)^{3} \beta^{3}\right. \\
& \quad+2 m n^{5}(1-\alpha)^{3} \alpha^{2} \beta+2 m^{5} n(1-\alpha)(1-\beta)^{2} \beta^{3} \\
& \quad+\frac{1}{3} m^{2} n^{4}(1-\alpha) \beta\left(3 \alpha^{2}(1-9 \beta)+2 \beta+3 \alpha^{2}(-1+5 \beta)+2 \alpha(-1+6 \beta)\right) \\
& \quad+\frac{4}{3} m^{3} n^{3}(1-\alpha) \beta\left(\beta(1+2 \beta)+\alpha^{2} \beta(-3+5 \beta)+\alpha\left(-1+3 \beta-7 \beta^{2}\right)\right) \\
& \left.\quad+\frac{1}{3} m^{4} n^{2}(1-\alpha) \beta\left(2 \beta\left(1+6 \beta-6 \beta^{2}\right)+\alpha\left(-2+3 \beta-18 \beta^{2}+15 \beta^{3}\right)\right)\right\} .
\end{aligned}
$$

\footnotetext{
${ }^{7}$ Here again it is possible to go to much higher orders.
} 


\section{Conclusion}

We have performed an important universality test of the conjectured quantum corrected string Bethe ansatz by demonstrating that the study of string sigma model one-loop corrections for rational three-spin strings uniquely determines the coefficients $\delta c_{r, s}$ to the same values as those found for the $\mathfrak{s l}(2)$ sector in $[27]^{8}$. Amazingly, despite the nonclosure of sub-sectors of the quantum string theory, a relatively simple dressing factor is capable of accounting for at least the lowest order quantum effects. While the explicit form of the one loop corrected dressing phase does not unambiguously explain the threeloop discrepancy between gauge theory and strings it does leave open the possibility that the discrepancy would be resolved by a full quantum string computation. Earlier ideas to explain the discrepancy via so-called gauge theory wrapping interactions now seem to be ruled out $[42,43]$. Obviously, we are in need for progress on the quantization of the string theory. Recently, an alternative formulation of a quantum string Bethe ansatz for certain sub-sectors has been proposed, based on an investigation of the $A d S_{5} \times S^{5}$ superstring in light cone gauge [44]. Furthermore, interesting works addressing the string quantization procedure via the study of various integrable sigma-models in two dimensions have appeared [45-47]. With our universality check we have provided evidence that any S-matrix resulting from such studies should reproduce not only the classical string Bethe ansatz but also include the one-loop correction term.

\section{Acknowledgments}

We thank N. Beisert for discussions. C.K. acknowledges the support of ENRAGE (European Network on Random Geometry), a Marie Curie Research Training Network supported by the European Community's Sixth Framework Programme, network contract MRTN-CT-2004-005616.

\section{References}

[1] J. A. Minahan and K. Zarembo. The Bethe-ansatz for $\mathcal{N}=4$ super Yang-Mills. JHEP, 03:013, 2003. hep-th/0212208.

[2] N. Beisert, C. Kristjansen, and M. Staudacher. The dilatation operator of $\mathcal{N}=4$ super Yang-Mills theory. Nucl. Phys., B664:131-184, 2003. hep-th/0303060.

[3] N. Beisert and M. Staudacher. The $\mathcal{N}=4$ SYM integrable super spin chain. Nucl. Phys., B670:439-463, 2003. hep-th/0307042.

[4] G. Mandal, N. V. Suryanarayana, and S.R. Wadia. Aspects of semiclassical strings in $A d S_{5}$. Phys. Lett., B543:81-88, 2002. hep-th/0206103.

\footnotetext{
${ }^{8}$ While this manuscript was beeing typed an interesting paper which gives another argument in favour of the conjectured quantum string Bethe ansatz appeared [41].
} 
[5] I. Bena, J. Polchinski, and R. Roiban. Hidden symmetries of the $A d S_{5} \times S^{5}$ superstring. Phys. Rev., D69:046002, 2004. hep-th/0305116.

[6] M. Staudacher. The factorized S-matrix of CFT/AdS. JHEP, 05:054, 2005. hepth/0412188.

[7] N. Beisert and M. Staudacher. Long-range $P S U(2,2 \mid 4)$ Bethe ansätze for gauge theory and strings. Nucl. Phys., B727:1-62, 2005. hep-th/0504190.

[8] N. Beisert and T. Klose. Long-range gl(n) integrable spin chains and plane-wave matrix theory. 2005. hep-th/0510124.

[9] L. Freyhult, C. Kristjansen, and T. Mansson. Integrable spin chains with $U(1)^{3}$ symmetry and generalized Lunin-Maldacena backgrounds. JHEP, 12:008, 2005. hep-th/0510221.

[10] G. Arutyunov, S. Frolov, and M. Staudacher. Bethe ansatz for quantum strings. JHEP, 10:016, 2004. hep-th/0406256.

[11] N. Beisert. The $s u(2 \mid 2)$ dynamic S-matrix. 2005. hep-th/0511082.

[12] R. A. Janik. The $A d S_{5} \times S^{5}$ superstring worldsheet S-matrix and crossing symmetry. 2006. hep-th/0603038.

[13] V. A. Kazakov, A. Marshakov, J. A. Minahan, and K. Zarembo. Classical / quantum integrability in AdS/CFT. JHEP, 05:024, 2004. hep-th/0402207.

[14] V. A. Kazakov and K. Zarembo. Classical / quantum integrability in non-compact sector of AdS/CFT. JHEP, 10:060, 2004. hep-th/0410105.

[15] N. Beisert, V.A. Kazakov, and K. Sakai. Algebraic curve for the $\mathrm{SO}(6)$ sector of AdS/CFT. 2004. hep-th/0410253.

[16] S. Schafer-Nameki. The algebraic curve of 1-loop planar $\mathcal{N}=4$ SYM. Nucl. Phys., B714:3-29, 2005. hep-th/0412254.

[17] N. Beisert, V. A. Kazakov, K. Sakai, and K. Zarembo. The algebraic curve of classical superstrings on $A d S_{5} \times S^{5}$. 2005. hep-th/0502226.

[18] N. Beisert, V. A. Kazakov, K. Sakai, and K. Zarembo. Complete spectrum of long operators in $\mathcal{N}=4$ SYM at one loop. JHEP, 07:030, 2005. hep-th/0503200.

[19] D. Berenstein, J.M. Maldacena, and H. Nastase. Strings in flat space and pp waves from $\mathcal{N}=4$ super Yang Mills. JHEP, 04:013, 2002. hep-th/0202021.

[20] C.G. Callan, H. K. Lee, T. McLoughlin, J. H. Schwarz, I. J. Swanson and X. Wu. Quantizing string theory in $A d S_{5} \times S^{5}$ : Beyond the pp- wave. Nucl. Phys., B673:340, 2003. hep-th/0307032. 
[21] C. G. Callan, T. McLoughlin and I. J. Swanson. Higher impurity AdS/CFT correspondence in the near-BMN limit. Nucl. Phys., B700:271-312, 2004. hepth/0405153.

[22] C. G. Callan, T. McLoughlin and I. J. Swanson. Holography beyond the Penrose limit. Nucl. Phys., B694:115-169, 2004. hep-th/0404007.

[23] D. Serban and M. Staudacher. Planar $\mathcal{N}=4$ gauge theory and the Inozemtsev long range spin chain. JHEP, 06:001, 2004. hep-th/0401057.

[24] Sakura Schafer-Nameki, Marija Zamaklar, and Konstantin Zarembo. Quantum corrections to spinning strings in $A d S_{5} \times S^{5}$ and Bethe ansatz: A comparative study. JHEP, 09:051, 2005. hep-th/0507189.

[25] S. Schafer-Nameki and M. Zamaklar. Stringy sums and corrections to the quantum string Bethe ansatz. JHEP, 10:044, 2005. hep-th/0509096.

[26] N. Beisert and A. A. Tseytlin. On quantum corrections to spinning strings and Bethe equations. Phys. Lett., B629:102-110, 2005. hep-th/0509084.

[27] R. Hernandez and E. Lopez. Quantum corrections to the string Bethe ansatz. 2006. hep-th/0603204.

[28] J. A. Minahan. Higher loops beyond the SU(2) sector. JHEP, 10:053, 2004. hepth/0405243.

[29] L. Freyhult and C. Kristjansen. Rational three-spin string duals and non-anomalous finite size effects. JHEP, 05:043, 2005. hep-th/0502122.

[30] M. Lubcke and K. Zarembo. Finite-size corrections to anomalous dimensions in $\mathcal{N}=4$ SYM theory. JHEP, 05:049, 2004. hep-th/0405055.

[31] N. Beisert, A. A. Tseytlin, and K. Zarembo. Matching quantum strings to quantum spins: One-loop vs. finite-size corrections. Nucl. Phys., B715:190-210, 2005. hepth/0502173.

[32] Rafael Hernandez, Esperanza Lopez, Africa Perianez, and German Sierra. Finite size effects in ferromagnetic spin chains and quantum corrections to classical strings. JHEP, 06:011, 2005. hep-th/0502188.

[33] I. Y. Park, A. Tirziu, and A. A. Tseytlin. Spinning strings in $A d S_{5} \times S^{5}$ : One-loop correction to energy in $S L(2)$ sector. JHEP, 03:013, 2005. hep-th/0501203.

[34] S. Frolov and A. A. Tseytlin. Multi-spin string solutions in $A d S_{5} \times S^{5}$. Nucl. Phys., B668:77-110, 2003. hep-th/0304255.

[35] S. Frolov and A. A. Tseytlin. Quantizing three-spin string solution in $A d S_{5} \times S^{5}$. JHEP, 07:016, 2003. hep-th/0306130. 
[36] S. A. Frolov, I. Y. Park, and A. A. Tseytlin. On one-loop correction to energy of spinning strings in $S^{5}$. Phys. Rev., D71:026006, 2005. hep-th/0408187.

[37] G. Arutyunov, J. Russo, and A. A. Tseytlin. Spinning strings in $A d S_{5} \times S^{5}$ : New integrable system relations. Phys. Rev., D69:086009, 2004. hep-th/0311004.

[38] Hiroyuki Fuji and Yuji Satoh. Quantum fluctuations of rotating strings in $A d S_{5} \times S^{5}$. 2005. hep-th/0504123.

[39] M. Blau, M. O'Loughlin, G. Papadopoulos, and A. A. Tseytlin. Solvable models of strings in homogeneous plane wave backgrounds. Nucl. Phys., B673:57-97, 2003. hep-th/0304198.

[40] S. Schafer-Nameki. Exact expressions for quantum corrections to spinning strings. 2006. hep-th/0602214.

[41] G. Arutyunov and S. Frolov. On $A d S_{5} \times S^{5}$ String S-matrix. 2006. hep-th/0604043.

[42] J. Ambjorn, R. A. Janik, and C. Kristjansen. Wrapping interactions and a new source of corrections to the spin-chain / string duality. Nucl. Phys., B736:288-301, 2006. hep-th/0510171.

[43] A. Rej, D. Serban, and M. Staudacher. Planar $\mathcal{N}=4$ gauge theory and the Hubbard model. 2005. hep-th/0512077.

[44] Sergey Frolov, Jan Plefka, and Marija Zamaklar. The $A d S_{5} \times S^{5}$ superstring in light-cone gauge and its Bethe equations. 2006. hep-th/0603008.

[45] N. Mann and J. Polchinski. Bethe ansatz for a quantum supercoset sigma model. Phys. Rev., D72:086002, 2005. hep-th/0508232.

[46] T. Klose and K. Zarembo. Bethe ansatz in stringy sigma models. 2006. hepth/0603039.

[47] N. Gromov, V. Kazakov, K. Sakai, and P. Vieira. Strings as multi-particle states of quantum sigma-models. 2006. hep-th/0603043. 